\title{
A Bio-Inspired AER Temporal Tri-Color Differentiator Pixel Array
}

\author{
Łukasz Farian, Member, IEEE, Juan A. Leñero-Bardallo, Member, IEEE, Philipp Häfliger, Senior Member, IEEE,
}

\begin{abstract}
This article investigates the potential of a bioinspired vision sensor with pixels that detect transients between three primary colors. The in-pixel color processing is inspired by the retinal color opponency that are found in mammalian retinas. Color transitions in a pixel are represented by voltage spikes, which are akin to a neuron's action potential. These spikes are conveyed off-chip by the Address Event Representation (AER) protocol. To achieve sensitivity to three different color spectra within the visual spectrum, each pixel has three stacked photodiodes at different depths in the silicon substrate. The sensor has been fabricated in the standard TSMC 90nm CMOS technology. A post-processing method to decode events into color transitions has been proposed and implemented as a custom interface to display real-time color changes in the visual scene. Experimental results are provided. Color transitions can be detected at high speed (up to $2.7 \mathrm{kHz}$ ). The sensor has a dynamic range of $58 \mathrm{~dB}$ and a power consumption of $22.5 \mathrm{~mW}$. This type of sensor can be of use in industrial, robotics, automotive and other applications where essential information is contained in transient emissions shifts within the visual spectra.
\end{abstract}

\section{INTRODUCTION}

Color enriches our visual world remarkably. The first steps in sensing color occurs in the retina. Thomas Young in the 19th century was the first to suggest that human color vision is trichromatic [1]. The trichromatic theory simply assumed that three images of the world were formed by these three sets of receptors and then transmitted to the brain where the ratios of the signals in each of the images were compared in order to sort out color appearances. That is roughly the operation that frame-based cameras with color filter perform to render color images. However, the idea of three images being transmitted to the brain is both inefficient and fails to explain several visually observed phenomena [2]. In fact, there is a complex retinal processing of the color information that is essential for our color perception.

Several authors have designed various artificial sensors trying to implement part of the retinal processing. The first bio-inspired vision sensor was presented by Fukushima [3] in the 1970s. Twenty years later Mahowald proposed the

J. A. Leñero-Bardallo has been partially supported by ONR project N0001414-1-0355, project MONDEGO (TEC2012-38921-C02-02) MINECO, project SMART CIS-3D (P12-TIC-2338), and project IPT-2011-1625-430000 MINECO.

Ł. Farian and P. Häfliger are with the Nanoelectronics Group, Department of Informatics, University of Oslo, Blindern 1072, Norway (e-mail lukaszf@ifi.uio.no; hafliger@ifi.uio.no).

J. A. Leñero-Bardallo is with the Institute of Microelectronics of Seville, CSIC-Universidad de Sevilla, Sevilla 41004, Spain (e-mail: juanle@imsecnm.csic.es).

Manuscript received January 15, 2015; revised September 11, 2015; accepted October 9, 2015. first integrated bio-inspired retina [4]. These sensors have been mainly focused on implementing the spatio-temporal processing performed in the human retina. More recently, several authors have started to pay attention to the color detection using bio-inspired vision sensors. However, to the best of our knowledge, the color processing made by retina has not been completely modeled with electronic circuits yet.

Conventional frame-based cameras usually place color filters over different pixels to render color images. Typically, a Bayer mask with filters centered at blue, green, and red is arranged over groups of four pixels. Then their outputs are combined to reconstruct color images. However, as previously stated, the retinal color processing is quite different. There are certain pairs of colors (opponents) that are mutually exclusive. Hering noted that certain hues were never perceived to occur simultaneously and he proposed an opponent-colors theory of color vision. These opponents are located in separated photoreceptors. In the human retina, there is a red/green opponency and a blue/yellow opponency. Ganglion cells receive inhibition or excitation from these different opponents that are always antagonistic [2].

Color detection has been traditionally neglected in bioinspired vision sensors. The main reason is that adding extra pixel processing decreases the fill factor and consequently the array resolution. Color filters require to have different pixels dedicated to detect each color. This is not something affordable when the array resolution is low. Recently, several authors have started to conceive the possibility of incorporating color processing with bio-inspired vision sensors [5]-[7]. This has been mainly motivated by the fact that modern fabrication technologies allow to stack photodiodes at different depths with different spectral sensitivity [7]-[9].

Color processing is quite important for some industrial processes, navigation systems, and robotics. For instance, it is essential to match samples of various colored materials or to operate mobile equipment for which the operating signals are colored lights [10]. In that sense, the good temporal resolution of bio-inspired cameras, their low latency and their low redundancy makes them good candidates for such applications.

In this article we present a bio-inspired vision sensor that can detect transient changes between primary colors at high speed. Changes between colors are detected by processing the photocurrent transient variations of three different stacked photodiodes with different spectral sensitivity. Temporal changes between three different colors are detected and opponencies between different colors in the retina are modeled. This work starts from our prior experience in processing color information with event-based color vision sensors [7]. In that work we 


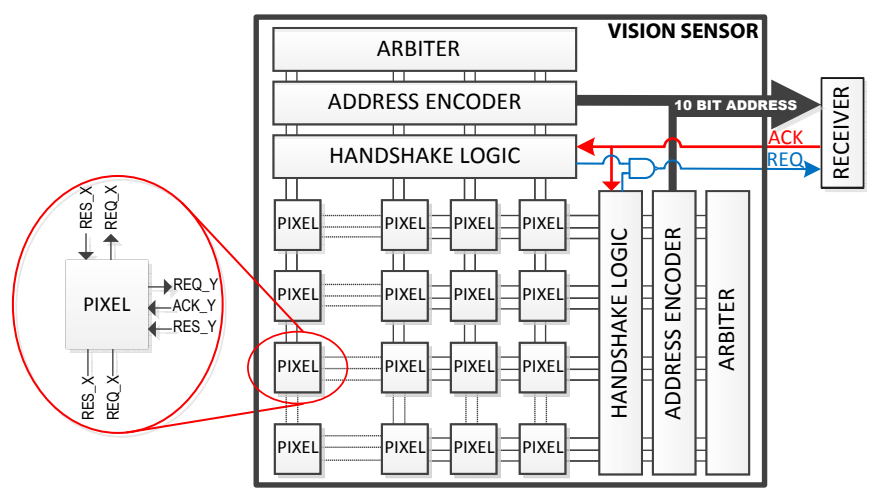

Fig. 1: Block level view of the pixel array and the AER communication periphery.

demonstrated that it was possible to render trichromatic images with event-based vision sensors and we proposed methods to sense and process the information coming from three stacked photodiodes. An octopus retina capable of generating RGB images was presented. With this work we aim to implement specific color processing conveyed in the retina, i.e. color temporal contrast detection. Moreover, some of our new work is inspired by the results previously published by Berner et al. [6]. Berner et al. designed a pixel that can detect transitions between two colors. With this new system, all transitions between the three primary colors can be detected. Furthermore, we have implemented a pixel array, and arbitration system, and programmed a dedicated interface to render real time images displaying the color transitions detected in the focal plane by the pixels that made up the pixel array. To the best of our knowledge, this is the first pixel array that can detect temporal color changes with an event-based bio-inspired vision sensor. Some preliminary results of this work were reported and displayed in BioCAS 2014 [11], [12]. In this invited paper, we provide a more detailed system description, a deeper insight into the system performance, more detailed and elaborated experimental results. Moreover, detailed descriptions of the experimental setup and the experiments conducted to characterize the sensor are provided.

This article is organized as follows: Section II explains the pixel design and the system description, including the AER arbiters. Section III describes the system experimental setup. Detailed experimental results of a single test pixel and the entire pixel array are provided. Finally, Section IV draws some conclusions and further system improvements.

\section{SYSTEM DESCRIPTION}

Fig. 1 displays the main system blocks. The color change temporal differentiator is made up of a $16 \times 16$ pixel matrix. Each pixel has three stacked photodiodes and some circuitry to detect temporal color changes. Pixels send out events when detecting transient color changes. Each pixel can generate four different kinds of events that indicate four different color transitions. For this reason, two extra bits are needed to encode the event type sent by each pixel. One bit of the event type bits is attached to the 4-bit column address, and one to the 4bit row address, these are multiplexed. Therefore five bits are

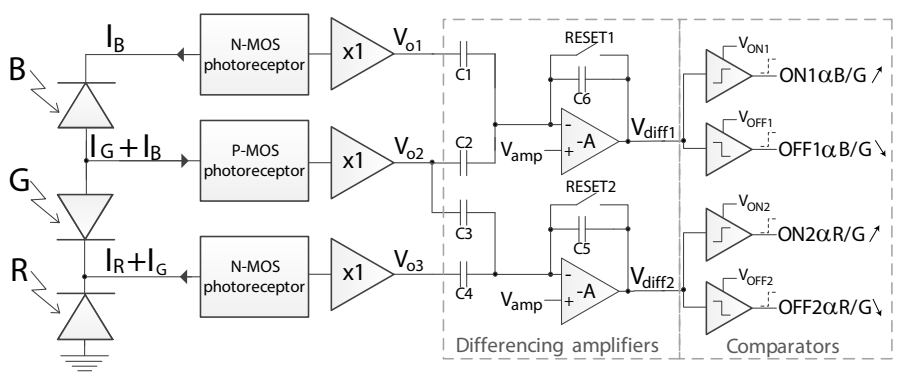

Fig. 2: Pixel core main blocks.

needed to encode the column addresses and five bits are needed to encode the row addresses. We used the arbitration scheme proposed by Häfliger in his $\mathrm{PhD}$ dissertation [13]. Such arbitration scheme is based on the one proposed by Boahen [14], with some modifications to add pipeline operation at the row operation. The circuitry has been previously implemented in other designs [7], [15] and can handle events rates up to $10 \mathrm{Meps}$ that is enough to arbitrate event rates from medium size pixel arrays. It is made up of some logic to handle the AER communication, multiplexers, and arbiters. In addition, a test pixel was included in the chip. The test pixel has several scan buffers connected to its internal nodes to measure their voltages as it will be depicted in the Experimental Results section.

\section{A. Pixel Design}

Fig. 2 shows the main pixel core constitutive blocks. The pixel design is inspired by the one proposed by Berner et al. [6] to detect color transitions. That one was based on the original one with switched capacitors proposed by Lichsteiner et al. [16]. The new pixel has been extended to operate with three stacked photodiodes and to handle the AER communication of a pixel matrix. Each pixel has three stacked photodiodes at different depths. Light penetration depth depends on photons wavelength [7]. Photons with higher energy (shorter wavelengths) travel less time though the crystal before generating electron-hole pairs. Hence, the top photodiode is more sensitive to blue light, and the bottom one more sensitive to longer wavelengths. For simplicity, let us denote $I_{B}, I_{G}$, and $I_{R}$ the photocurrents through the top, the middle, and the bottom photodiodes. Combination of these currents go to logarithmic photoreceptors whose outputs are voltages that depend on their input photocurrents. The next stage consists of two different amplifiers that amplify transient variations of their input voltages. Inputs to the amplifiers are buffered by source followers. Amplifiers are followed by several comparators that send out spikes. Finally there is some logic to handle the AER communication that is also included in the pixel and will be explained afterwards.

Fig. 3 depicts the logarithmic photoreceptors circuitry and how the two kind of photoreceptors are connected to the stacked photodiodes. Since the photocurrents flowing through the photodiodes have different directions, an N-MOS version and PMOS version of the photoreceptor have to be used depending if the photocurrents sink or go out of the photoreceptors. The 


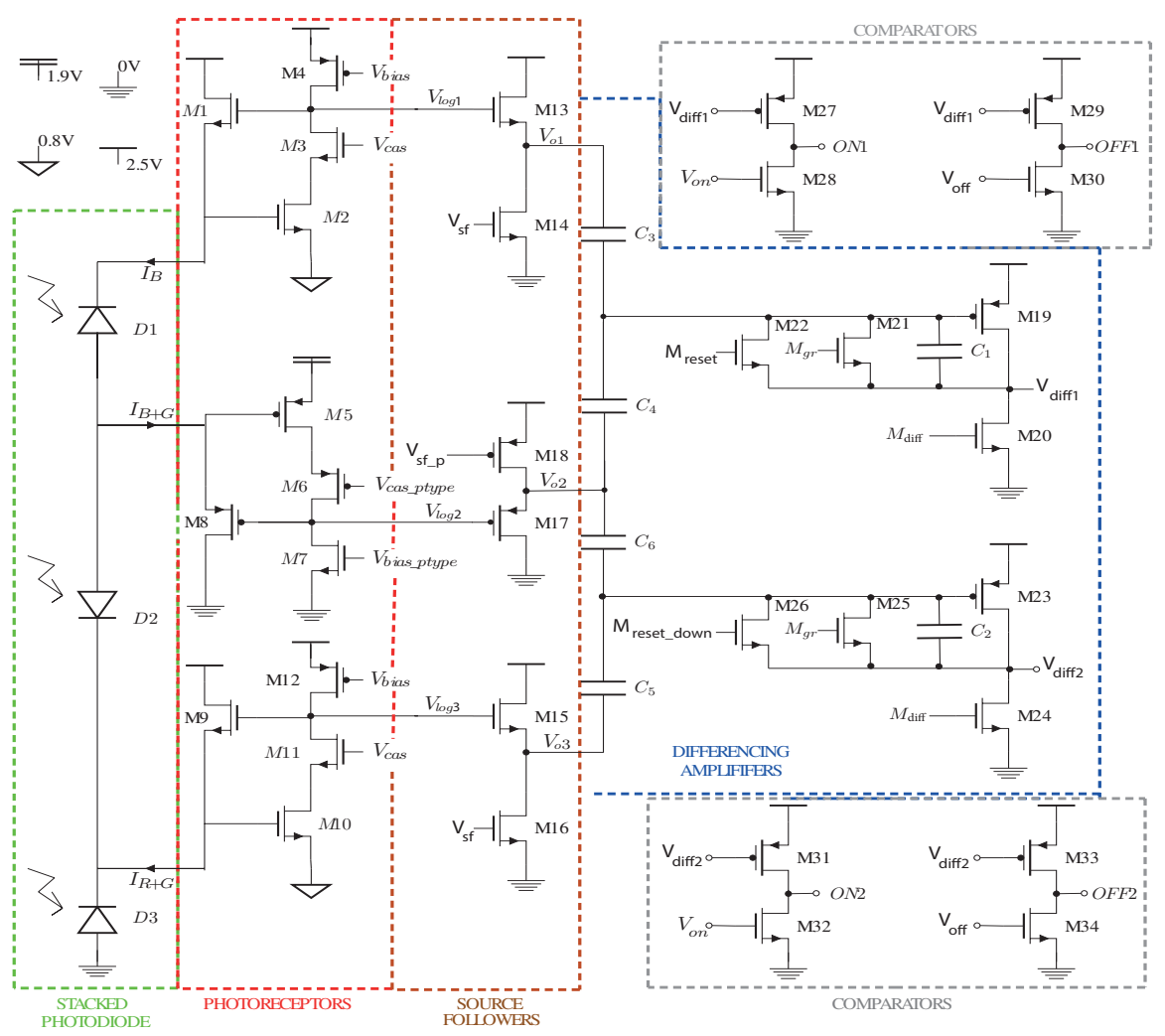

Fig. 3: Detailed pixel core schematics. It includes logarithmic photoreceptors, source followers, differencing amplifiers, and comparators. Transistor sizes are: $(W / L) \mu m / \mu m M_{1}=M_{9}=0.4 / 0.5, M_{8}=0.4 / 2, M_{2}=M_{5}=M_{10}=1 / 0.5$, $M_{3}=M_{6}=M_{11}=2 / 0.4, M_{4}=M_{7}=M_{12}=0.5 / 5, M_{13}=M_{14}=M_{15}=M_{16}=0.6 / 0.3, M_{17}=0.5 / 1.6$, $M_{18}=1.2 / 0.4, M_{19}=M_{23}=M_{27}=M_{29}=M_{31}=M_{33}=1.1 / 0.6, M_{20}=M_{24}=M_{28}=M_{30}=M_{32}=M_{34}=1 / 2.3$. Nominal bias voltages: $V_{\text {bias }}=1.7 \mathrm{~V}, V_{\text {bias_ptype }}=0.7 \mathrm{~V}, V_{\text {cas }}=1.6 \mathrm{~V}, V_{\text {cas_ptype }}=1 \mathrm{~V}, V_{s f}=0.7 \mathrm{~V}, V_{s f \_p}=1.9 \mathrm{~V}$, $M_{\text {diff }}=1.1 \mathrm{~V}, V_{\text {on }}=1 \mathrm{~V}, V_{\text {off }}=1.2 \mathrm{~V}$.

outputs of three logarithmic photoreceptors are:

$$
\begin{array}{r}
V_{\log _{1}}=U_{T} \frac{1}{\kappa_{1}} \ln \left(\frac{I_{B}}{I_{s_{1}}}\right)+V_{T} \\
V_{\log _{2}}=-U_{T} \frac{1}{\kappa_{8}} \ln \left(\frac{I_{B}+I_{G}}{I_{s_{8}}}\right)+V_{T} \\
V_{\log _{3}}=U_{T} \frac{1}{\kappa_{9}} \ln \left(\frac{I_{G}+I_{R}}{I_{s_{9}}}\right)+V_{T}
\end{array}
$$

Please note that the P-MOS version of the photoreceptor has negative gain. The voltage variations at the source followers outputs will be:

$$
\begin{array}{r}
\Delta V_{o 1}=U_{T} \frac{\kappa_{13}}{\kappa_{1}} \Delta \ln \left(\frac{I_{B}}{I_{s_{1}}}\right) \\
\Delta V_{o 2}=-U_{T} \frac{\kappa_{17}}{\kappa_{8}} \Delta \ln \left(\frac{I_{B}+I_{G}}{I_{s_{8}}}\right) \\
\Delta V_{o 3}=U_{T} \frac{\kappa_{15}}{\kappa_{9}} \Delta \ln \left(\frac{I_{G}+I_{R}}{I_{s_{9}}}\right)
\end{array}
$$

Source followers are necessary to drive the output voltages to the differencing amplifiers that have a high input capacitive load. The output voltage variations at the differencing amplifiers outputs can be approximated as:

$$
\Delta V_{\text {diff }} \approx-\frac{C_{i n}}{C_{\text {out }}} \Delta V_{\text {in }}=-A \Delta V_{\text {in }}
$$

The input voltages $V_{o 1}-V_{o 2}$ and $V_{o 3}-V_{o 2}$ of Equations (4)(5)-(6) will be amplified by the differencing amplifiers:

$$
\begin{array}{r}
\Delta V_{d i f f_{1}}=-A_{1} U_{T} \frac{\kappa_{13}}{\kappa_{1}} \Delta \ln \left(\frac{I_{B}}{I_{s_{1}}}\right) \\
+A_{2} U_{T} \frac{\kappa_{17}}{\kappa_{8}} \Delta \ln \left(\frac{I_{B}+I_{G}}{I_{s_{8}}}\right) \\
\Delta V_{d i f f_{2}}=-A_{3} U_{T} \frac{\kappa_{15}}{\kappa_{9}} \Delta \ln \left(\frac{I_{B}+I_{G}}{I_{s_{9}}}\right) \\
+A_{4} U_{T} \frac{\kappa_{17}}{\kappa_{8}} \Delta \ln \left(\frac{I_{G}+I_{R}}{I_{s_{9}}}\right)
\end{array}
$$

For simplicity, we can assume that all the $\kappa_{*}$ coefficients values are the same. The capacitors values have been chosen the same to obtain identical gain values, i.e. $A_{1}=A_{2}=$ $A_{3}=A_{4}=C_{3} / C_{1}=C_{4} / C_{1}=C_{5} / C_{2}=C_{6} / C_{2}$. Hence, the previous equations can be simplified: 


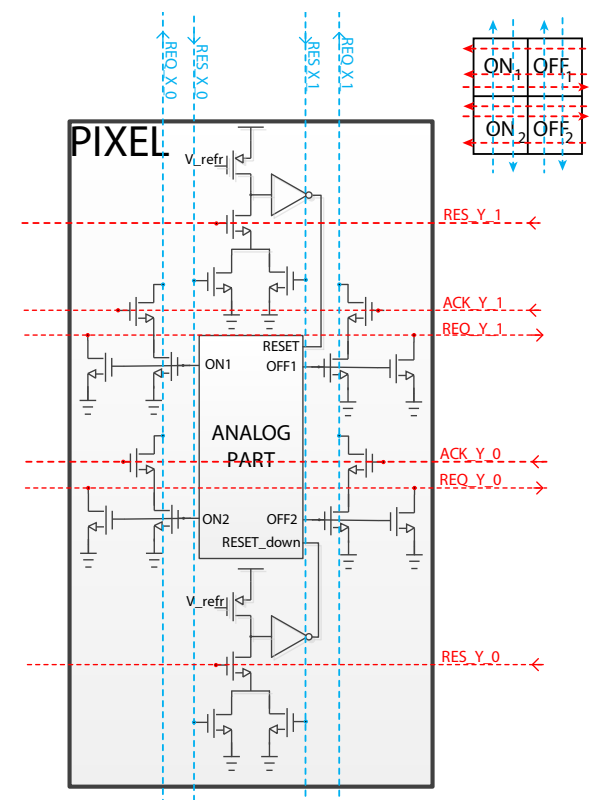

Fig. 4: Complete pixel block diagram including AER logic. Analog part is described in Fig. 3.

$$
\begin{aligned}
& \Delta V_{d i f f_{1}}=-A U_{T} \Delta \ln \left(\frac{I_{B}}{I_{B}+I_{G}}\right) \\
& \Delta V_{d i f f_{2}}=-A U_{T} \Delta \ln \left(\frac{I_{G}+I_{R}}{I_{B}+I_{G}}\right)
\end{aligned}
$$

Examining Equations (10) and (11), we can deduce that a negative increment of $V_{\text {diff }}$ can be interpreted as a shift to the blue and positive increment means a shift to the green in the spectrum. A negative increment of $V_{\text {diff } f_{2}}$ can be interpreted as a shift to the red/near infrared and positive increment means a shift to the green in the spectrum. Amplifiers outputs are connected to comparators with adjustable thresholds monitoring whether $V_{d i f f *}$ has increased or decreased. Hence, four different event types can be sent out by the pixel: $O N_{1}, O F F_{1}$, $\mathrm{ON}_{2}, \mathrm{OFF}_{2}$, as it is displayed in Fig. 3 .

In Fig. 3 the area of the gate of the PMOS transistor M8 is smaller compared to NMOS transistors M1 and M9 in order to compensate the difference between $\kappa_{8}$ and $\kappa_{1 / 9}$. The slope factor for the transistors operating in subthreshold region is determined by $n=1 / \kappa=1+C_{j 0} / C_{o x}$, where $C_{o x}$ is a gate capacitance and $C_{j 0}$ is a junction capacitance. Due to the higher doping level in n-well compared to PSUB, the depletion capacitance $C_{j 0}$ for the PMOS transistor is higher. Based on $C_{j 0}=W L \sqrt{q \epsilon_{0} N_{\text {sub }} /\left(4 \Phi_{F}\right)}$, by reducing the area of PMOS transistor M8 the junction capacitance decreases, which results in similar values of $\kappa_{8}$ and $\kappa_{1 / 9}{ }^{1}$.

\section{B. Pixel AER logic}

Pixels use Address Event Representation (AER) protocol to send out spikes when detecting color changes [13]. To

\footnotetext{
${ }^{1} \mathrm{q}$ is electrical charge of the electron, $\epsilon_{0}$ is absolute permittivity, $N_{s u b}$ is channel doping and $\Phi_{F}$ is Fermi potential.
}

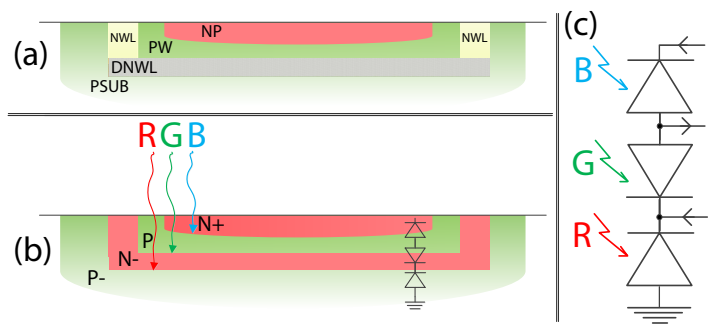

Fig. 5: Stacked photodiodes cross section diagram. (a) Design layers. (b) n-p junctions. (c) Stacked photodiodes diagram.

handle the AER communication, some logic circuitry have to be added to the pixel as it is displayed in Fig. 4. Pixels can generate four different event types $\left(O N_{1}, O F F_{1}, O N_{2}\right.$, $\mathrm{OFF}_{2}$ ). For arbitration purposes, each pixel can be considered as an array of four pixels arranged in a $2 \times 2$ pixel matrix as it is depicted on the top-right corner of Fig. 4. Two bits are used to encode the different four event types. The X- and Yaddresses have five bits. Four of them are dedicated to encode the pixel column/row number and the other one contains event type information. Therefore, logic to handle the AER communication has to be replicated twice. Every time that the input signals $R E S_{-} X_{-*}$ and $R E S_{-} Y_{-*}$ are active simultaneously, the corresponding differencing amplifier that has elicited one spike is reset. Note that one differencing amplifier cannot generate $O N_{*}$ and $O F F_{*}$ events simultaneously. The voltage $V_{r e f r}$ is a tunable pull up voltage that controls the rise time of the reset pulses. This rise time is called a refractory time. As it is in real neurons, a refractory time is a silent time between consecutive spikes of the same pixel. It is useful to limit pixels activity.

\section{Stacked Photodiodes}

Some modern CMOS fabrication technologies offer the possibility of fabricating a deep n-well to isolate the substrate of p- or n-MOS transistors. This n-well diffusion can be also used to create two buried photodiodes that can also sense photons. If we combine a n-well diffusion with a deep nwell diffusion as is shown in Fig. 5, it is possible to stack three different photodiodes in silicon. Each one will have different spectral sensitivity. The top one will have a peak of sensitivity closer to the blue, the middle one will have more sensitivity close to the green, and the bottom one will be more sensitive to light in the red/NIR band. Unfortunately, the depth of the different $p+/ n+$ junctions is not disclosed by the foundry and cannot be chosen by the designer. We will refer in the paper to the photodiodes spectra as B, G, and $\mathrm{R}$. Incident radiations shifts within the visual spectra will cause transient photocurrent variations that will be detected by the pixels processing circuitry. We assume that spectral ratios are equivalent to measured photodiodes current ratios. Table I depicts how different $O N_{*}$ and $O F F_{*}$ events are translated into spectral ratio variations and color changes.

\section{EXPERIMENTAL RESULTS}

A $16 \times 16$ pixel array was fabricated in the standard TSMC 90nm CMOS process. A custom PCB with an attached $4 \mathrm{~mm}$ 
TABLE I: Translation of $O N_{*}, O F F_{*}$ events into color changes.

\begin{tabular}{ccc}
\hline Event & Spectra ratio & Color change \\
\hline$O N_{1}$ & $\propto \frac{B}{G} \nearrow$ & bluer \\
$O F F_{1}$ & $\propto \frac{B}{G} \searrow$ & greener \\
$O N_{2}$ & $\propto \frac{R}{G} \nearrow$ & redder \\
$O F F_{2}$ & $\propto \frac{R}{G} \searrow$ & greener \\
\hline
\end{tabular}
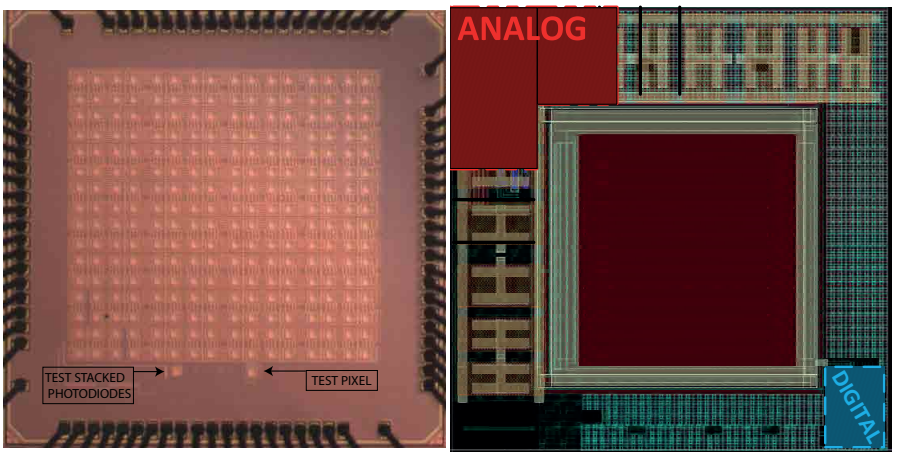

Fig. 6: Left: Chip microphotograph. Right: Pixel layout. Chip dimensions are $1.3 \mathrm{~mm} \times 1.3 \mathrm{~mm}$. Pixel dimensions are $82 \mu \mathrm{m}$ x $82 \mu \mathrm{m}$.

$F / 1.2 \mathrm{C}$-mount lens was designed to test the system. The AER data from the vision sensor was logged by the USBAERmini2 board [17] and then this data was sent through a USB port to a PC. A custom Java interface was adapted to show real time color transitions [18]. Main system specifications are summarized in Table II. Chip dimensions are $1.3 \mathrm{~mm} \times$ $1.3 \mathrm{~mm}$. Fig. 6 shows a chip microphotograph (left) and the pixel layout of a single pixel (right). In addiction to the $16 \times 16$ pixel matrix, the pixel and its arbitration system, two test structures were included in the chip: a set of three stacked photodiodes and a test pixel with several analog scan buffers

TABLE II: Chip Specifications

\begin{tabular}{|c|c|}
\hline \hline Fabrication process & TSMC 90nm CMOS \\
\hline Power Supply & $2.5 \mathrm{~V}$ \\
\hline Type & Temporal color contrast detection \\
\hline Color transitions detected & $\mathrm{R} \leftrightarrow \mathrm{G} \leftrightarrow \mathrm{B}$ \\
\hline Interface & $\mathrm{AER}$ \\
\hline Pixel size & $42 \mu \mathrm{m} \times 82 \mu \mathrm{m}$ \\
\hline Photodiode size & $27 \%$ \\
\hline Fill factor & 58 transistors, $6 \mathrm{MIM}$ capacitors \\
\hline Pixel complexity & $16 \mathrm{x} 16$ \\
\hline Array size & $1.3 \mathrm{~mm} \times 1.3 \mathrm{~mm}$ \\
\hline Die size & $9-19 \mathrm{~mA} @ 2.5 \mathrm{~V}$ \\
\hline Power consumption & $58 \mathrm{~dB}$ \\
\hline Dynamic Range & $40 \mathrm{~dB}$ \\
\hline Intra-Scene & \\
\hline Dynamic Range & $2.7 \mathrm{kHz}$ \\
\hline Pixel bandwidth & \\
\hline \multicolumn{2}{|c}{} \\
\hline \hline
\end{tabular}

TABLE III: Test LED Specifications

\begin{tabular}{|c|c|c|c|}
\hline \hline & Min. Wavelength & Typ. Wavelength & Max. Wavelength \\
\hline Red & $618 \mathrm{~nm}$ & $625 \mathrm{~nm}$ & $630 \mathrm{~nm}$ \\
\hline Green & $520 \mathrm{~nm}$ & $525 \mathrm{~nm}$ & $535 \mathrm{~nm}$ \\
\hline Blue & $455 \mathrm{~nm}$ & $460 \mathrm{~nm}$ & $465 \mathrm{~nm}$ \\
\hline \hline
\end{tabular}

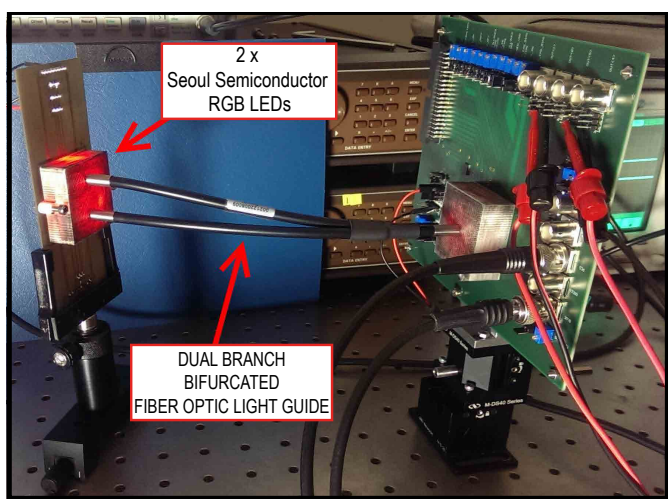

Fig. 7: Experimental setup to test a single pixel response to different color changes. Light from a RGB LEDs is transmitted with a fiber cable.

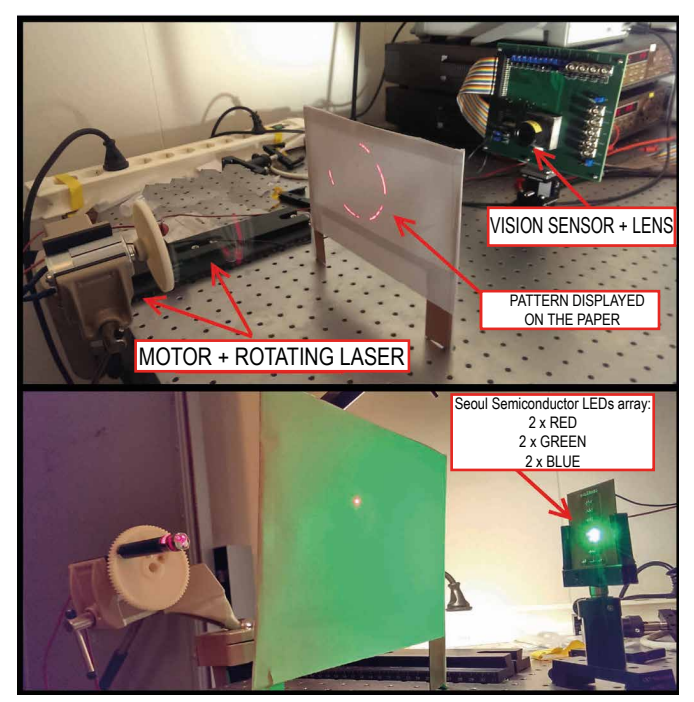

Fig. 8: Experimental setup to test the pixel array with different color stimuli moving over different color backgrounds.

as it is shown in Fig. 6 left.

\section{A. Experimental Setup}

We describe here the different experimental setups for the different measurements that will be explained in the next subsections.

To characterize the photodiodes spectral sensitivity (see Subsection III-B), the chip was illuminated with the BPS101 Tungsten Halogen Light Source with different color filters, centered at different wavelengths ranging between $400 \mathrm{~nm}$ and $950 \mathrm{~nm}$. The sensitivity measurements, light source irradiance for different wavelengths, filters attenuation factors and bandwidths (50nm or $80 \mathrm{~nm}$, depending on the filter) were taken into account. In order to characterize DC responses of logarithmic photoreceptors, the BPS101 Tungsten Halogen Light Source with different attached neutral density filters was used (see Subsection III-C1). To measure the color change sensitivity of the test pixel (see Subsection III-C2) and the pixel bandwidth (see Subsection III-C4), a test bench to dynamically change input colors was built. Three high-power full 


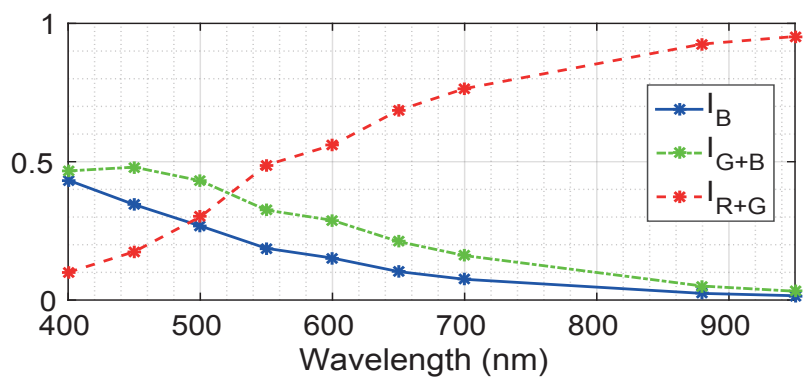

(a) Measured photocurrents relative values.

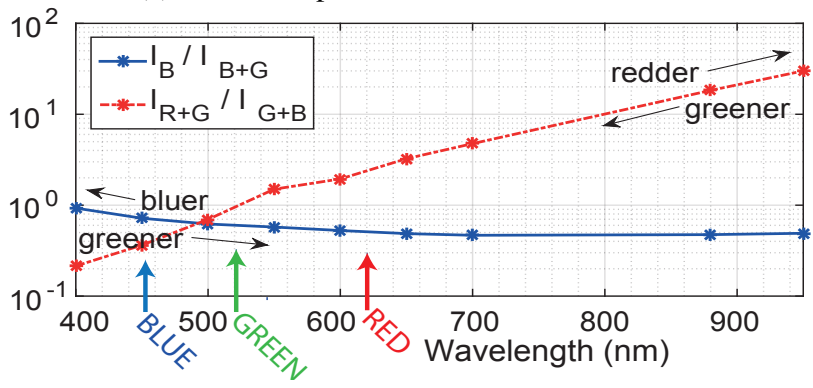

(b) Computed Photocurrents ratios.

Fig. 9: Spectral response of three stacked photo diodes in TSMC 90nm technology. The values were corrected by the Halogen Light Source irradiance factor and color filters transmission efficiency.

color LEDs F50360 from Seoul Semiconductor emitting light at different wavelengths were available for the experiments (see diodes specifications on Table III). In the test bench shown in Fig. 7, two LEDs were used simultaneously to introduce controlled transient color changes. Light from two separate RGB diodes was mixed and transmitted by a dual branch light guide (Dolan-Jenner E809). Two input branches of the light guide were attached to the PCB with individual RGB diodes. The output branch was attached to the PCB with the sensor. The light intensity of the diodes was controlled by a waveform generator. Such solution enabled to vary different colors ratios of emitted light in order to measure the pixel sensitivity to changes in colors ratios.

To determine the sensor matrix array response to moving color stimuli (see Subsections III-D1 and III-D3), the experimental setup of Fig. 8 was set. The vision sensor was stimulated by a beam of a collimated light (strong light dot) over different color backgrounds. The background color was projected by another PCB equipped with six single color LEDs (two red, two green, and two blue SZB05A0A diodes). Such solution provided scattered light suitable to project a background illumination.

\section{B. Photodiodes Spectral Response}

As we explained previously, the photodiodes depths are not disclosed by the foundry. For this reason, the photodiodes design was conservative $(41.5 \mu \mathrm{m} \times 43.4 \mu \mathrm{m})$ and not optimized in terms of area consumption. Fig. 9a shows the measured spectral response of the three photodiodes normalized with the sum of the three photocurrents that we measured. Photodiodes
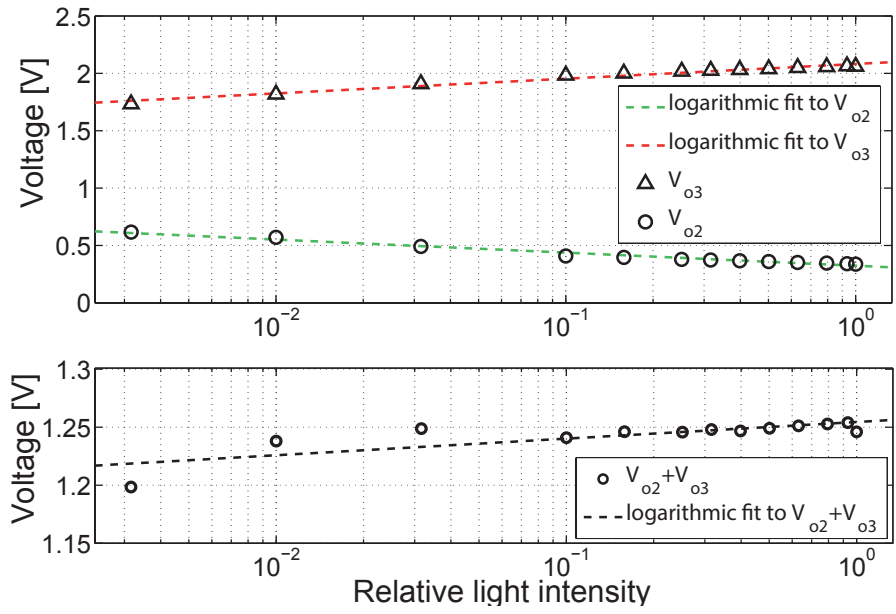

Fig. 10: Experimental measurement of DC responses of logarithmic photoreceptors. Different combinations of the Newport metallic neutral density filters were used: $(0.03,0.1,0.3,1,2$, 3)OD. Top: outputs $V_{o 2}$ and $V_{o 3}$. Bottom: sum $V_{o 2}+V_{o 3}$.

have different quantum efficiencies and different junction capacitances that are not known accurately. As we were expecting, the top one is the least sensitive to light, and the bottom one is the most sensitive. The bottom one has higher quantum efficiency and the size of its depletion region is bigger than others. Note that we are not interested in photocurrent absolute values to detect color transitions. The current ratio variations is what is processed to detect color changes. Fig. $9 \mathrm{~b}$ displays the computed values of the two current ratios (see Equations (10) and (11)), whose variations provoke events that indicate color transitions and illustrates how these ratios can be used to discriminate between colors. As we discussed previously, photocurrent ratios are proportional to spectral ratios. The $\mathrm{R} / \mathrm{G}$ ratio increases when the color spectrum shifts towards red, and $\mathrm{B} / \mathrm{G}$ ratio decreases when the color spectrum shifts towards blue. The ratio B/G exhibits weaker dependence on color spectra, it has steepest slope up to $650 \mathrm{~nm}$ and after that is flat, while the $\mathrm{R} / \mathrm{G}$ ratio has almost constant slope over the entire tested spectrum. The ratio $\mathrm{B} / \mathrm{G}$ exhibits weak dependence on longer spectrum wavelengths because both the top and the middle photodiode are least sensitive in this region. Possibly the top and the middle photodiodes are located much shallower than the third one, so only very energetic photons can be caught by these photodiodes. Thus, if a narrow band light source moves from $600 \mathrm{~nm}$ to $400 \mathrm{~nm}$ wavelength it would clearly lead to positive B/G events and negative R/G events as it is summarized in Table I. Inversely, if a narrow band light source moves from $600 \mathrm{~nm}$ to $900 \mathrm{~nm}$ wavelengths, it would lead to positive R/G events. Eventually, it would also lead to a few negative $\mathrm{G} / \mathrm{B}$ events.

\section{Pixel Performance}

1) Logarithmic response: The sensor was illuminated by the coherent flat spectrum light beam. Different combinations of the Newport metallic neutral density filters of different transmittance were placed between the light source and the 


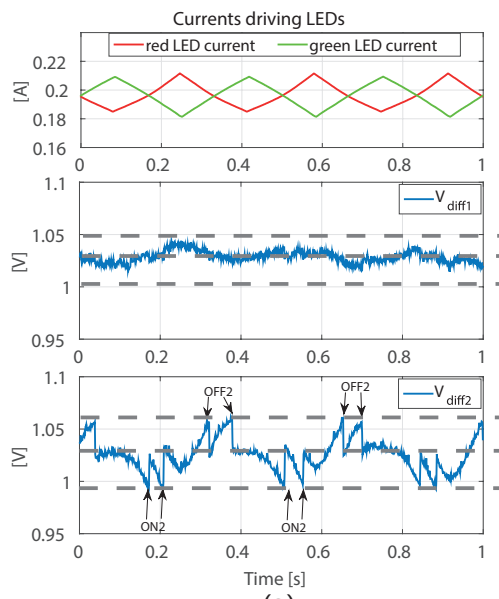

(a)

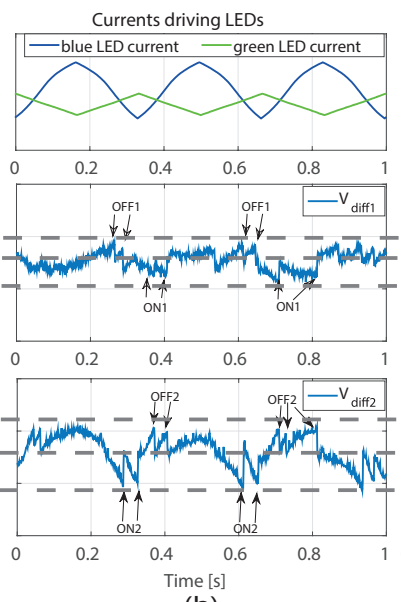

(b)

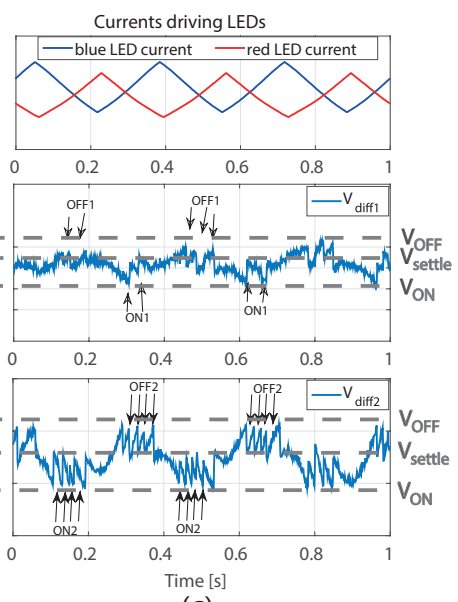

(c)

Fig. 11: Pixel response to the continuous: (a) red-green color change, (b) blue-red color change, (c) blue-green color change. The intensity of red and green LEDs light was swept approximately one decade from 800lux to 8klux, whereas the blue LED light was swept 1.5 decade in order to compensate the lower sensitivity of the top photodiode.

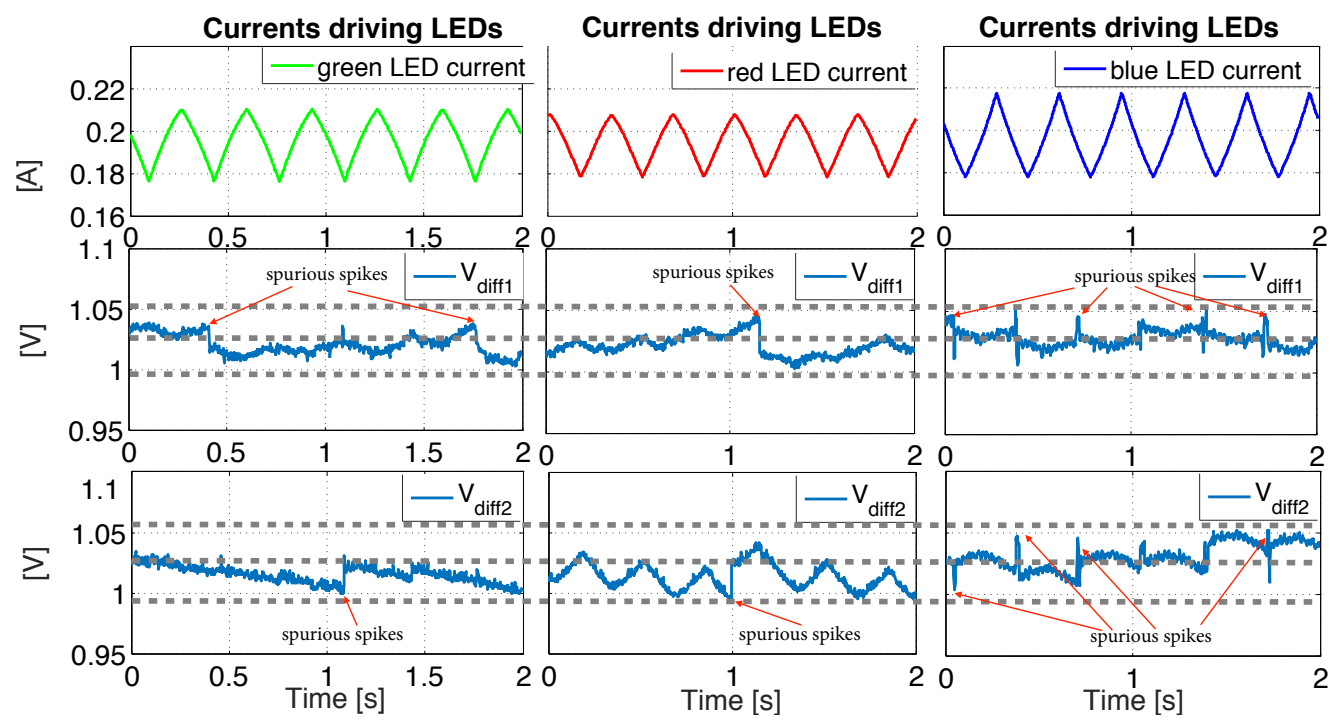

Fig. 12: Pixel response to light intensity change, without changing the spectral color. The LEDs intensity was swept within similar range as in Fig. 11.

camera. The outputs of logarithmic photoreceptors from Fig. 3 were measured with scan buffers included on chip. The resulting responses are shown in Fig. 10 (top). Voltage $V_{o 2}$ is the output of the P-MOS version of the logarithmic photoreceptor and voltage $V_{o 3}$ is the output of the N-MOS photoreceptor. Tested photoreceptors perform logarithmic amplification over the entire measurement range. They have opposite gains because P-MOS photoreceptor sinks and N-MOS photoreceptor drives the photocurrents.

Both logarithmic photoreceptors responded oppositely to a light transient intensity change. Ideally, if both signal pathways had the same gains, the sum $V_{o 2}+V_{o 3}$ would give a flat line. In bottom plot in Fig. 10 we observed a change of the sum approximately $50 \mathrm{mV}$ over 3 decades light intensity change. In section System Description, we discussed how to size the PMOS transistor $M_{8}$ and NMOS transistors $M_{1} / M_{9}$ so the difference between their slope factors was minimized as the simulation results suggested. However, the bottom plot in Fig. 10 suggests that slope factor in subthreshold region in TSMC90nm CMOS process is not accurately modeled, as it was also reported for another process by Berner et al. [6].

2) Color Changes Detection: We characterized the pixel response to different color intensity changes and compared these responses with the assumed translations from Table I.

First, we measured the pixel response to a continuous redgreen intensity change by illuminating its surface with $3 \mathrm{~Hz}$ triangular out-of-phase red and green light beams. The $V_{O N_{x}}$ voltage thresholds were set to $V_{O N_{x}}=0.99 \mathrm{~V}$ and $V_{O F F_{x}}=$ $1.06 \mathrm{~V}$. Top plots in Fig. 11 show the currents through the LEDs and remaining plots show the $V_{\text {dif } f_{*}}$ voltages responses of a single test pixel to such stimuli. $V_{\text {settle }}$ is the differencing amplifiers output voltage when reseting them. As 
shown in Fig. 11.a, transient shifts between the $R$ to the $G$ spectra provoke events when variations of the output voltage $V_{\text {diff } 2}$, representing the R/G spectra ratios, exceeds the voltage thresholds $V_{\mathrm{ON}_{2}}$ and $V_{O F F_{2}}$. We observed roughly four $O N_{2} / O F F_{2}$ events. We did not observe $O N_{1} / O F F_{1}$ events from the variations of the second photocurrent ratio $I_{B} / I_{B+G}$. Ideally, with no blue light change stimulus we would expect strong variations of $V_{\text {diff } 1}$. However, as shown in Fig. 9a, since the photodiodes have not narrowband spectra, the top photodiode also responds to green light, resulting in the same proportional change of the nominator and the denominator of the photocurrents ratio $I_{B} / I_{B+G}$. The transient shifts between the B to the G spectra (Fig. 11.(b)) provoked four types of events. Events $O N_{1}$ occur for the positive increment of blue light (B/G increases) and conversely, $O F F_{1}$ events occur for the positive increment of green light (B/G ratio decreases). Besides that, we received a similar number of $O F F_{2}$ and blue $\mathrm{ON}_{2}$ events. blue The current driving the blue LED in the most upper plot of Fig. 11b had higher range compared to other currents because the top photodiode has lower quantum efficiency than the rest of the photodiodes. The shape of the current driving the blue LED did not follow the triangular shape because the circuit controlling the brightness of the LED was providing almost its maximum output current for this experiment. Finally, for the continuous blue-red contrast changes (Fig. 11.c), we observed the same response scheme as for the spectra shifting between B and $G$, but with higher variations of the output voltage $V_{d i f f 2}$, and the moderate activity of the output voltage $V_{\text {diff } 1}$. The output voltage $V_{\text {diff } 2}$ exhibits high activity for spectra shifting between B and $\mathrm{R}$ because the change coefficient $\Delta\left(I_{R} / I_{G}\right)$ is higher for this case as shown in Fig. $9 \mathrm{~b}$

The comparator thresholds $O N_{*}$ and $O F F_{*}$ were set identical because they are set and determined by identical comparators. However, the plots in Fig. 11 suggest that the events generated by $V_{\text {diff } 1}$ are triggered for lower thresholds than for $V_{d i f f 2}$. The reason for this is the mismatch between two analog buffers which bring voltage outputs $V_{\text {diff } 1}$ and $V_{\text {diff } 2}$ to the output pads.

The final observations conclude that pixels are more sensitive to $\mathrm{R} / \mathrm{G}$ variations rather than $\mathrm{B} / \mathrm{G}$, because, as it was shown in Fig. $9 b$, the $R / G$ ratio has much steeper slope than $B / G$ ratio for color changes. For the color transitions between $R$ and $G$ spectra, only R/G events are elicited (Fig. 11.a). On the other hand, if the color transitions between spectra involve shorter wavelengths (Fig. 11.b and c) we obtain both R/G and B/G types of events out of phase, e.g. positive $R / G$ events always follow negative $\mathrm{B} / \mathrm{G}$ events and vice versa.

3) Light Intensity Change: Fig. 12 shows the pixel response to an intensity change. We modulated the intensity of only one LED and the other one was turned off, so only the light intensity was modulated, without changing the spectral color. The LEDs intensity was swept within similar range as in Fig. 11. We kept the same $V_{O N_{x}}$ and $V_{O F F_{x}}$ voltage thresholds as in the previous test. The fluctuations of the output voltages $V_{\text {diff } 1}$ and $V_{\text {diff } 2}$ for the red and green light intensity changes are due to the nonidentical gains in signal pathways $V_{o 1}, V_{o 2}$ and $V_{o 3}$. Recording events during 2 seconds, we

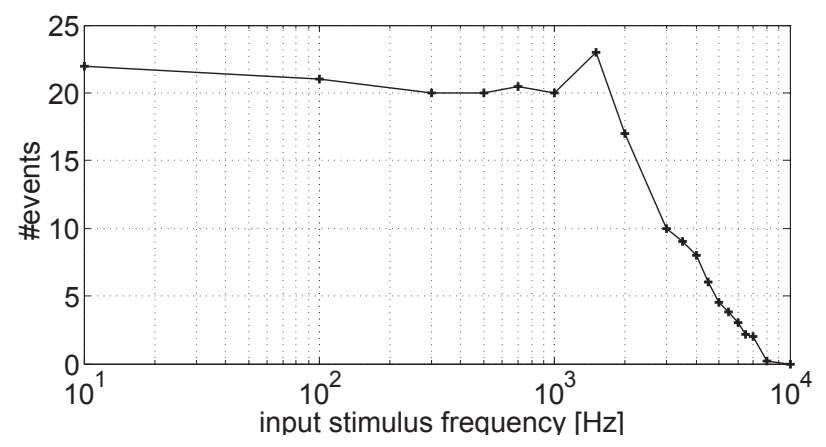

Fig. 13: Pixel frequency response. A test pixel was illuminated simultaneously with a uniform green background light and with a red LED light modulated with a sinusoidal signal. The number of counted events divided by number of sine wave cycles is plotted for different sinusoidal frequency values.

observed $1-2$ spurious spikes when stimulating with the red and green LEDs. We received 4-5 spurious spikes for the blue color intensity variations, what is more than for the two other colors intensity variations. This behavior can be explained by the low sensitivity of the stacked photodiodes to short wavelengths. The blue color does not penetrate through the substrate sufficiently and does not generate a sufficient amount of photocurrent in all three photodiodes. As a result, the assumptions of the logarithmic gains in Eq. 10 and Eq. 11 are not always valid and the pixel does not operate properly.

4) Pixel Bandwidth: The test pixel was stimulated by a sinusoidal red LED light of 1 klux brightness with a green LED generating a constant background. The bias values were set to optimize speed of the differencing amplifiers and the refractory period was set much smaller than the all stimuli period values in the experiment to ensure it did not affect the bandwidth measurements. Events $\mathrm{ON}_{2} / O F F_{2}$ were counted for 20 cycles for the stimulus frequency ranging from $10 \mathrm{~Hz}$ to $10 \mathrm{kHz}$ and results were divided by the number of stimulus cycles. The pixel has a second order response as is shown in Fig. 13 with a resonance peak about $1.5 \mathrm{kHz}$ before the response rolls off. For the frequency value of $2.7 \mathrm{kHz}$ the measured pixel sensitivity is reduced to the half, what is equivalent to the pixel bandwidth.

\section{Matrix Array Performance}

1) Focal Plane Color Changes Detection: The remaining tests were performed on the entire pixel matrix with a rotating intensive light dot (beam of a collimated light) over different color backgrounds. For different stimuli the sensor generated asynchronous pixel events that were conveyed off-chip by the Address Event Representation (AER) protocol and recorded. Then we used this recorded data to reconstruct space-temporal images, as shown in Fig. 14. Fig. 14(a) shows the response to a rotating green dot over a red background, Fig. 14(b) shows the response to a rotating green dot over a blue background, and Fig. 14(c) shows a rotating red dot over a blue background. Rotating dots and the background illumination were generated by the narrow band photodiodes described in Section III-A. 

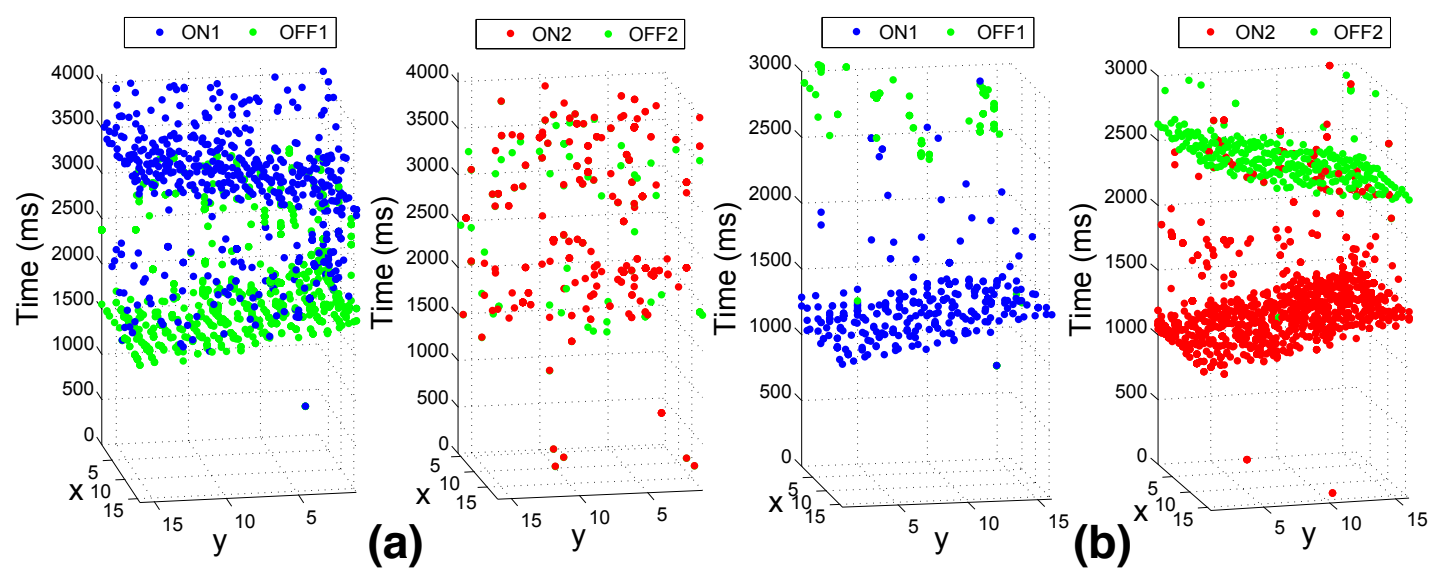

Fig. 15: Space-time plots showing pixel responses to: (a) color transition blue $\rightarrow$ green $\rightarrow$ blue, (b) color transition green $\rightarrow$ red $\rightarrow$ green. Color edges were printed on a white paper and shifted in front of the camera.

Comparing Fig. 14(a) with Fig. 14(b-c), we observed that for the first case (green dot over on a red background) much more events representing changes of the $\mathrm{R} / \mathrm{G}$ ratio are registered. We can identify the green-red transitions: green events precede while red events follow the stimulus. The pattern in Fig. 14a) is similar to the one from Fig.11, where we obtained much more ON2/OFF2 events. Spurious events ON1/OFF1 are most probably elicited by the crosstalk between ON2/OFF2 and ON1/OFF1 events types. The tests in Fig. 14(b) and Fig. 14(c) which involve shifts between blue and green, and between blue and red spectrum, are similar. In both cases more events representing changes of the $\mathrm{B} / \mathrm{G}$ ratio are elicited than $\mathrm{R} / \mathrm{G}$ ratio, meaning that if there is a blue color spectrum involved in color transitions, ON2/OFF2 events are dominating. This indicates that the camera is capable of detecting transitions between adjacent primary color spectra, e.g. $\mathrm{R} \leftrightarrow \mathrm{G}$ and $\mathrm{B} \leftrightarrow \mathrm{G}$, rather than $\mathrm{R} \leftrightarrow \mathrm{B}$ directly. Three snapshots with different timestamps illustrating the live color transitions of the corresponding tests are shown in Fig. 14. In order to discriminate between red and blue spectra, we decoded $O N_{1}$ and $O F F_{1}$ events as a transitions between adjacent spectra. In other words, based on how many events have been accumulated, $O N_{1}$ events are decoded as a color transitions from red through green and finally to blue, and inversely $O F F_{1}$, events are decoded as a color transitions from blue through green to red. Finally, events $O N_{2}$ and $O F F_{2}$ were decoded, as initially assumed in Table I, as a shift between red and green colors. Fig. 16(a) illustrates the decoding process of the events into live color transitions. Every time that an event is received, the color associated to each pixel is updated and stored. Combining this information with the incoming events, the color transitions between primary colors are detected and displayed. Fig. 16(b) displays how events are received within a possible scenario of transient color transitions. It should be noted that for this design, there is no circuit implementation which can directly compare transitions between $\mathrm{B} / \mathrm{R}$ spectra. The proposed approach, based on analyzing the resulting events, does not provide direct discrimination between $B / R$ spectra, but it provides a possibility to track changes between adjacent spectral regions.

In another experiment, we moved paper sheets with printed blue-green and red-green discontinuities in front of the vision sensor following the direction of the arrows plotted in Fig. 15. We measured the responses to blue $\rightarrow$ green $\rightarrow$ blue and green $\rightarrow$ red $\rightarrow$ green color transitions, as shown in Fig. 15(a) and Fig. 15(b) respectively. Surface slopes in Fig. 15 indicate how fast the color edges were moved through the visual field of the camera. It can be estimated that the entire displacement took approximately $0.5 \mathrm{~s}$. Pixels activity was limited by the refractory time to limit the number of events $1-2$ events per edge with such strong color transitions.

2) Uniformity of Response: In order to quantify the uniformity of the response, a gradual blue->green->blue transition was swept in front of the entire visual field of the sensor. The test was repeated 30 times for 4 different thresholds between $\pm 50 \mathrm{mV}$ and $\pm 150 \mathrm{mV}$. All $O N_{1}$ and $O F F_{1}$ events were counted and used to calculate the distribution of the events per pass of a single color transition and corresponding fitted gaussian distributions, shown in Fig. 17. For the highest threshold we observed on average of $1.3 O N_{1}$ and $1.2 O F F_{1}$ events per the transition and for the lowest: $21.2 O N_{1}$ and 18.2 $O F F_{1}$ events. Similarly as reported in [16] we can observe that the width of the distributions is inversely proportional to the thresholds, indicating that for small settings of thresholds, the comparators mismatch becomes dominating.

3) Dynamic Range: We tested the sensor intra-scene dynamic range by monitoring the camera response to the movement of the partly shaded rotating colorful disc of Fig. 18(a). We used yellow color instead of green in order to provoke larger variations of $\mathrm{B} / \mathrm{G}$ ratio. As we explained previously in Section III-B, the top and the middle photodiodes have similar spectral sensitivity, the ratio $\mathrm{B} / \mathrm{G}$ exhibits weaker dependence on color spectra shifts than the R/G ratio. Yellow-blue shifts in the spectrum are easier to distinguish because yellow color spectrum contains primary colors with longer wavelengths towards red. The disc was exposed to the light of illuminance of $60 \mathrm{klux}$. A neutral density filter of 2 decades attenuation was placed in front of the sensor covering partly the visual field 
TABLE IV: State-of-the-art comparison.

\begin{tabular}{|c|c|c|c|c|c|c|}
\hline Work & $\begin{array}{ll}\text { Graaf } & 1997 \\
{[19]} & \end{array}$ & $\begin{array}{ll}\text { Graaf } & 1998 \\
\text { [9] } & \end{array}$ & $\begin{array}{l}\text { Olsson } \\
2008[5]\end{array}$ & $\begin{array}{l}\text { Berner } 2011 \\
{[6]}\end{array}$ & $\begin{array}{l}\text { Juanle } 2013 \\
\text { [7] }\end{array}$ & This work \\
\hline Functionality & $\begin{array}{l}\text { PDM } \\
\text { Synchronous } \\
\text { Pixel } \\
\text { (BIFET } \\
\text { process) }\end{array}$ & $\begin{array}{l}\text { Synchronous } \\
\text { Pixel with } \\
\text { in-Pixel A/D } \\
\text { Conversion }\end{array}$ & $\begin{array}{l}\text { PDM Color } \\
\text { Asyn- } \\
\text { chronous } \\
\text { Event Pixel }\end{array}$ & $\begin{array}{l}\text { Dynamic } \\
\text { Color } \\
\text { Vision } \\
\text { Sensor } \\
\text { Pixel }\end{array}$ & $\begin{array}{l}\text { PDM/TFS } \\
\text { Color Asyn- } \\
\text { chronous } \\
\text { Event } \\
\text { Sensor }\end{array}$ & $\begin{array}{l}\text { Dynamic } \\
\text { Color } \\
\text { Vision } \\
\text { Sensor }\end{array}$ \\
\hline $\begin{array}{l}\text { Number } \\
\text { of Spectral } \\
\text { Regions }\end{array}$ & Dichromatic & Dichromatic & Dichromatic & Dichromatic & Trichromatic & Trichromatic \\
\hline Resolution & $\begin{array}{l}1 \text { pixel (2 } \\
\text { stacked } \\
\text { photodiodes) }\end{array}$ & $\begin{array}{l}1 \text { pixel (2 } \\
\text { stacked } \\
\text { photodiodes) }\end{array}$ & $\begin{array}{l}1 \quad \text { pixel }(2 \\
\text { stacked } \\
\text { photodi- } \\
\text { odes) }\end{array}$ & $\begin{array}{l}1 \quad \text { pixel }(2 \\
\text { stacked } \\
\text { photodi- } \\
\text { odes) }\end{array}$ & $\begin{array}{l}24 \quad \times \quad 24 \\
(3 \quad \text { stacked } \\
\text { photodi- } \\
\text { odes })\end{array}$ & $\begin{array}{l}16 \quad \times \quad 16 \\
(3 \quad \text { stacked } \\
\text { photodi- } \\
\text { odes })\end{array}$ \\
\hline $\begin{array}{lr}\text { Pixel } & \text { Size } \\
\text { and } & \text { Fill } \\
\text { Factor } & \\
\end{array}$ & $\begin{array}{l}400 \mu \mathrm{m} \quad \times \\
400 \mu \mathrm{m}, \mathrm{ND}\end{array}$ & $\begin{array}{l}\text { Photodiode: } \\
800 \mu \mathrm{m} \times \\
600 \mu \mathrm{m}, \mathrm{ND}\end{array}$ & $\begin{array}{l}1188 \mu^{2} \\
8.74 \%\end{array}$ & $\begin{array}{l}29 \mu \mathrm{m}, \times \\
29 \mu \mathrm{m}, 17 \%\end{array}$ & $\begin{array}{l}31 \mu \mathrm{m}, \quad \times \\
31 \mu \mathrm{m}, 28 \%\end{array}$ & $\begin{array}{l}82 \mu \mathrm{m}, \quad \times \\
82 \mu \mathrm{m}, 27 \%\end{array}$ \\
\hline $\begin{array}{l}\text { Dynamic } \\
\text { Range }\end{array}$ & $>60 \mathrm{~dB}$ & $80 \mathrm{~dB}$ & ND & $>80 \mathrm{~dB}$ & $>60 \mathrm{~dB}$ & $\begin{array}{l}58 \mathrm{~dB}(40 \mathrm{~dB} \\
\text { intra-scene) }\end{array}$ \\
\hline $\begin{array}{l}\text { Bandwidth } \\
\text { or Output } \\
\text { Frequency }\end{array}$ & $20-80 \mathrm{kHz}$ & $0.2-20 \mathrm{kHz}$ & $0-35$ eps & $2 \mathrm{kHz}$ & 0.1-6Meps & $2.7 \mathrm{kHz}$ \\
\hline
\end{tabular}

of the camera to create an illumination step. We show four different snapshots at different timestamps. We can recognize in the images four edges: one red, one blue and two green. The top green edge at the time of $0 \mathrm{~ms}$ indicates a shift from blue to green spectrum and the bottom green edge indicates a shift from red to green spectrum. The blue edge is tailing because the photoreceptor bandwidth is proportional to photocurrent, [20]. This experiment proves that the vision sensor has an intra-scene dynamic range of $40 \mathrm{~dB}$.

The same experimental setup with a rotating colorful disc was used to test the camera dynamic range, but this time we provided uniform light conditions across the visual filed of the camera. The camera was able to discriminate colors for the illumination conditions ranging from 120lux up to 100klux, thus the camera has a dynamic range of $58 d B$

4) Temporal Noise: A static uniform white scene was set in front of the vision sensor and all possible sources of unwanted noise, as flickering light bulbs, computer displays, were turned off. For such uniform and noiseless scene conditions we recorded undesired spurious $O N_{*}$ and $O F F_{*}$ events within time of $50 \mathrm{~s}$ and then we used these results to compute the average event rate. Fig. 19 shows the pixels average event rate and their standard deviation as a function of chip illuminance. Error bars in Fig. 19 top show the maximum and minimum pixel values. Some pixels have an ideal behavior and did not fire. This undesired pixels activity is a result of noise sources in the circuit and it increases inversely proportionally to the light intensity because the power spectral density noise spectrum at the output of differencing amplifier $V_{d i f f *}$ is, according Berner et al. [6]:

$$
S_{o}(f) \approx \frac{8 A^{2} U_{T}^{2} q}{I_{p h}} \cdot \frac{1}{1+\left(f / f_{0}\right)^{2}}
$$

Where A is the gain of the differencing amplifier, $I_{p h}$ is the input photocurrent, and $f_{0}$ is the $3 \mathrm{~dB}$ cutoff frequency of the differencing amplifier. For illumination conditions below 0.1lux this noise provokes many undesired events which dominate, so the contrast recognition becomes not possible any more, what stablished the lowest value of illumination where the sensor can operate. For higher illumination values, we did not experience any limitation in the sensor operation.

\section{E. Power consumption}

The chip power consumption (excluding pads, and analog and digital buffers of the test pixel) varies between $9-19 \mathrm{~mA}$, depending on bias settings and the event rate. The power consumption could be reduced in further designs by resizing the differencing amplifier transistors of Fig. 3. The differencing amplifiers are implemented as common-source amplifiers with a quiescent current flowing through transistors $M_{19}, M_{20}$, and $M_{23}, M_{24}$. This quiescent current is dominant source of power consumption of the pixel and the entire matrix. We managed to limit the quiescent current, and hence decrease the power consumption, by lowering a default bias voltage $M_{\text {diff }}$. Since lowering of the bias voltage would cause $V_{d i f f *}$ to hit the rail, another solution to limit the power consumption of the sensor is to decrease the conductances of transistors $M_{19}, M_{20}, M_{23}$, $M_{24}$.

\section{F. Benchmarking and comparison to the state-of-the-art}

Table IV compares the features and performance of our work with previously reported contributions with pixels having stacked photodiodes to achieve spectral sensitivity. We have classified the sensors according to their functionality, i.e. color intensity detection or color temporal contrast detection. Graaf et al. proposed a synchronous pixel with two stacked photodiodes in 1997 implemented in BIFET technology [19]. Later on, an improved version in CMOS technology was presented by the same authors, [9]. It was a single synchronous sensor with one pixel and A/D conversion. After these pioneering contributions, Olsson et al. proposed a concept of bio-inspired event-based vision pixel capable of differentiating two spectral regions, [5]. It was an octopus pixel. In 2011, Berner et al. presented a color dynamic vision pixel to detect 

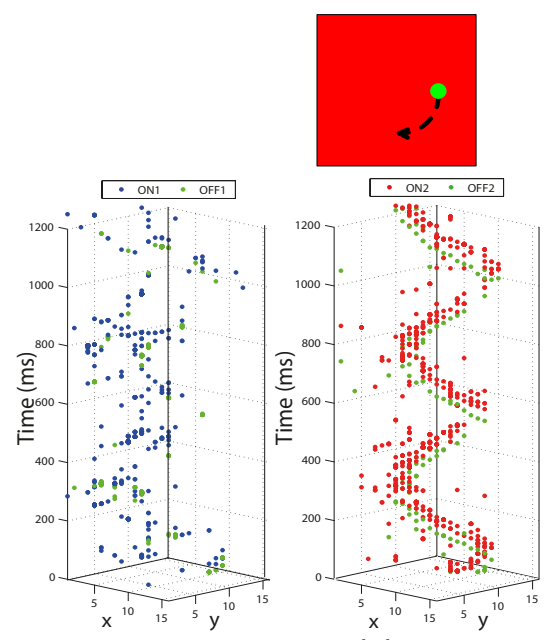

(a)
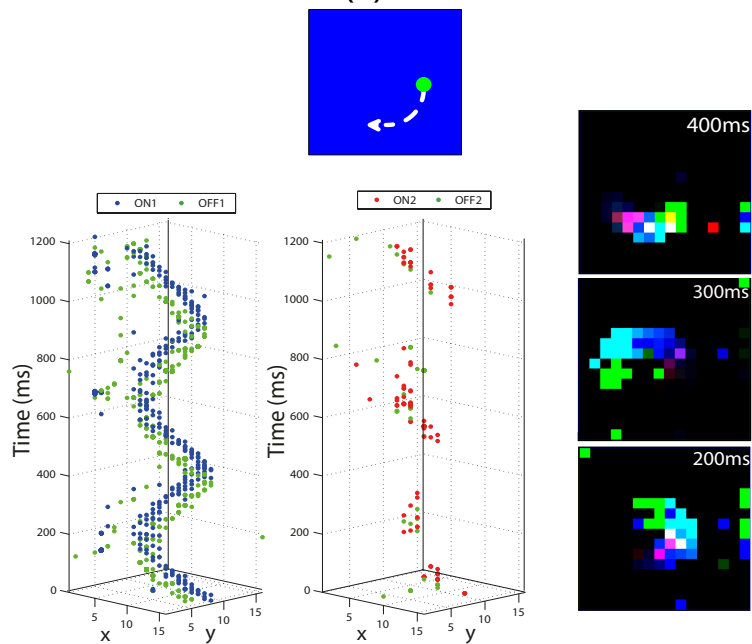

(b)
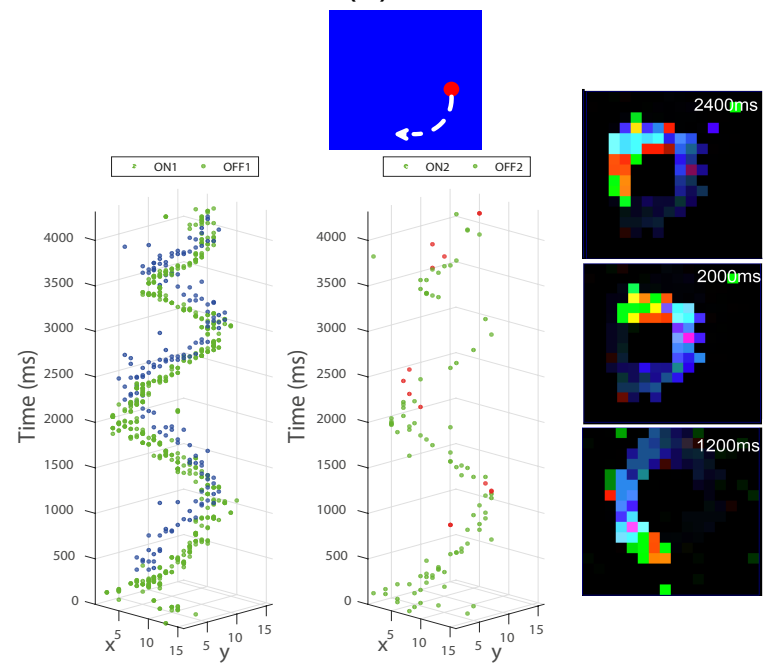

(c)

Fig. 14: Space-time plots (left) and snapshots from a live image (right) show the response to: (a) a rotating green dot over red background $\left(\# O N_{1}=951, \# O F F_{1}=811\right.$, $\# O N_{2}=3048, \# O F F_{2}=1819$ events in 1.2s), (b) a rotating green dot over blue background ( $\# O N_{1}=649$, \#OF $F_{1}=$ 1241, $\# O N_{2}=127, \# O F F_{2}=85$ events in $1.2 \mathrm{~s}$ ), (c) a rotating red dot over blue background $\left(\# O N_{1}=332\right.$, $\# O F F_{1}=1531, \# O N_{2}=14, \# O F F_{2}=104$ events in $4.0 \mathrm{~s})$.
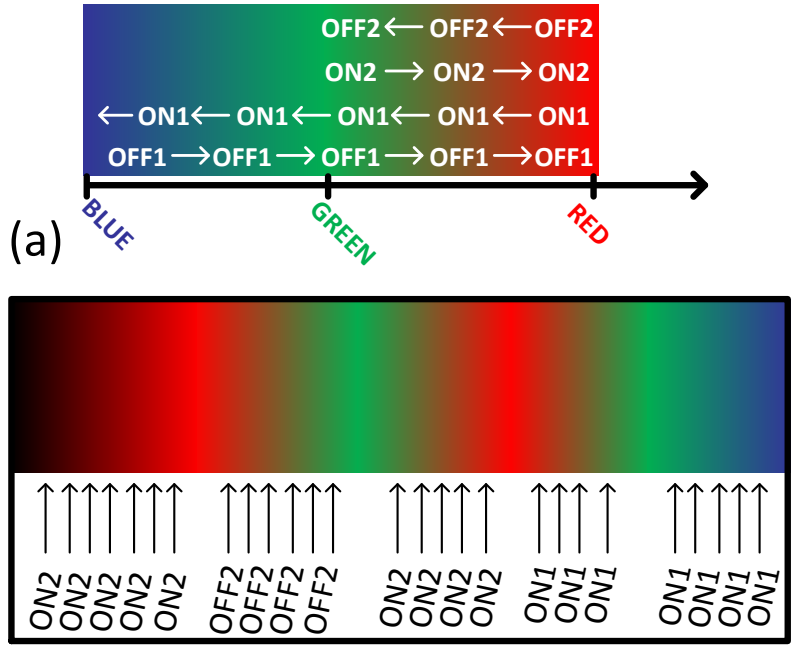

(b)

Fig. 16: a) Illustration how the events from the pixels are decoded into live color transitions. b) Possible scenario of color transitions. Every time that $O N_{1}$ events are received the pixel color shifts to shorter wavelengths.

temporal transitions between two spectral regions [6]. It was the first bio-inspired pixel capable of doing some in-pixel color processing. Afterwards, the authors initiate a research line based on incorporating stacked diodes with three $\mathrm{p} / \mathrm{n}$ junctions to discriminate three spectral regions within the visible spectrum and render tri-color images closer to the human perception. The first AER color pixel array was an octopus retina capable of rendering RGB intensity images with PDM (Pulse Density Modulation) or TFS (Time-to-firstspike) readouts [7]. Based on this work and the remarkable contribution of Berner et al. [6], we decided to adapt the pixel circuitry to detect transient transitions between primary colors. With this new work we also give light to solve technical questions like the pixel array testing and how to interpret and reconstruct the temporal contrast information provided by three different stacked photodiodes. We have programmed a Java interface to process the incoming events and display real-time images that represent transient color transitions. In theory, any event-based processing already implemented for monochromatic DVSs could be extended to be used with our system. To do so, once a color transition is detected, the same processing conducted with a monochromatic DVS should be implemented. Note that our system responds only to shifts in the spectrum, not to the light intensity changes. Hence, it would be possible to distinguish the color of the different regions of one image, but not their intensity. To achieve color quality in the image reconstruction, it would be necessary to have narrowband spectra photodiodes with the accurate control of the depth of the different $\mathrm{p} / \mathrm{n}$ junctions. Observing the works reported in Table IV, we believe that further AER color vision sensors will incorporate more than a single pixel and operate in several spectral regions. 


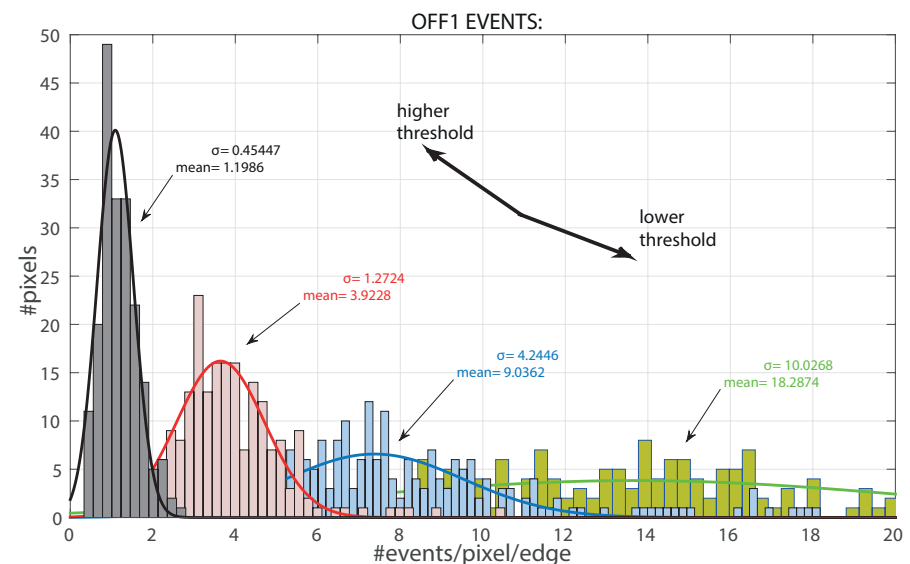

(a)

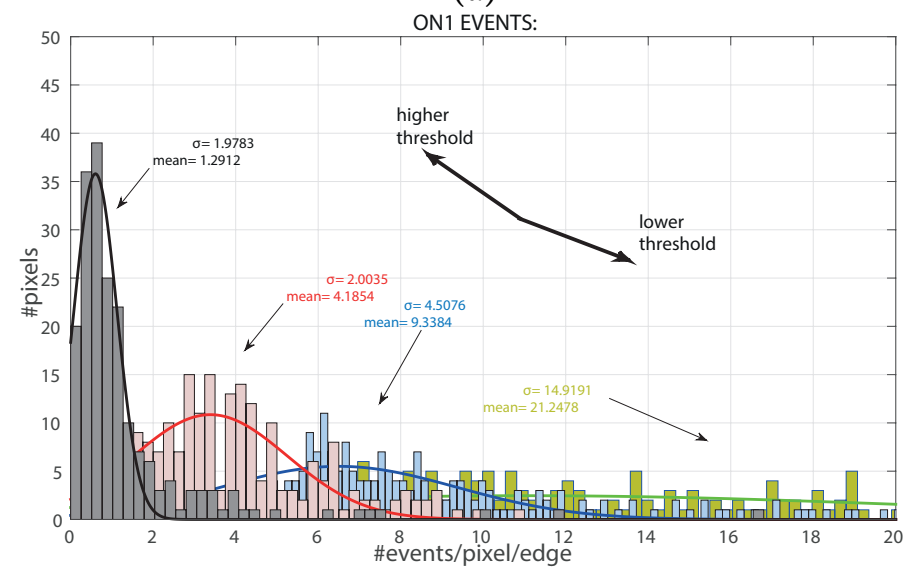

(b)

Fig. 17: Vision sensor test of the uniformity of the response. Histograms show the distribution of $O F F_{1}$ (a) and $O N_{1}$ (b) events per pass of a blue->green->blue gradual transition for 30 repetitions in front of the visual field of the sensor. For the highest threshold we observed on average of $1.3 O N_{1}$ and 1.2 $O F F_{1}$ events per the transition and for the lowest: $21.2 O N_{1}$ and 18.2OFF 1 events. The thresholds $V_{O N_{X}}$ and $V_{O F F_{X}}$ were set between $\pm 50 \mathrm{mV}$ and $\pm 150 \mathrm{mV}$.

\section{CONCLUSIONS}

We built and tested the first focal plain array of bio-inspired AER pixels that mimic color opponencies among primary colors in the retina. Its pixels are capable of detecting fast transient color changes between adjacent primary colors (up to $2.7 \mathrm{kHz}$ ). We proposed a post-processing method to decode events into color transitions between nonadjacent color spectra $(\mathrm{R} \leftrightarrow \mathrm{B})$ and implemented an interface displaying these events as real-time color changes. Detailed experimental results from a single test pixel and from a pixel array are reported. Since the vision sensor abandons a global integration time and each pixel communicates asynchronously whenever it detects temporal color contrast, the post processing load on a CPU, based on color codes or colored object outlines, is relaxed. This vision sensor has good potential for an automotive robotics applications where high speed temporal color contrast recognition, especially of fast moving objects in different lighting conditions, is demanded. According to our experimental re-

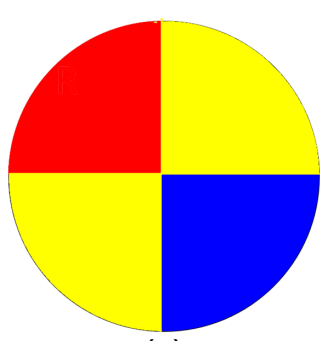

(a)

Oms

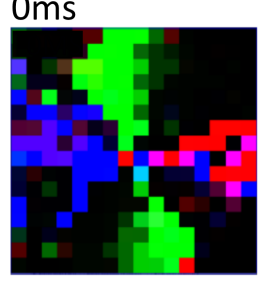

$24 \mathrm{~ms}$

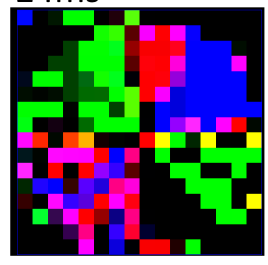

(c)

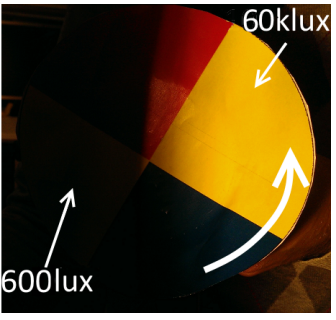

(b)

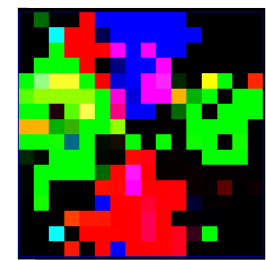

$38 \mathrm{~ms}$

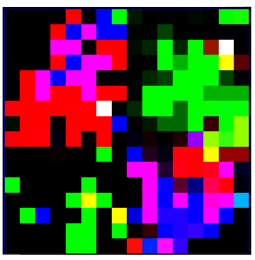
$12 \mathrm{~ms}$

Fig. 18: Test of vision sensor intra-scene dynamic range. A rotating disc painted red-yellow-blue-yellow was placed in front of the camera (a), half of the disc was exposed to lower illumination (b). The darker part was exposed to the light of irradiance of $600 \operatorname{lux}$, the rest of the disc was exposed to a source irradiance of 60klux. (c) Several snapshots taken at different timestamps

. Disk rotation frequency was 20rps.
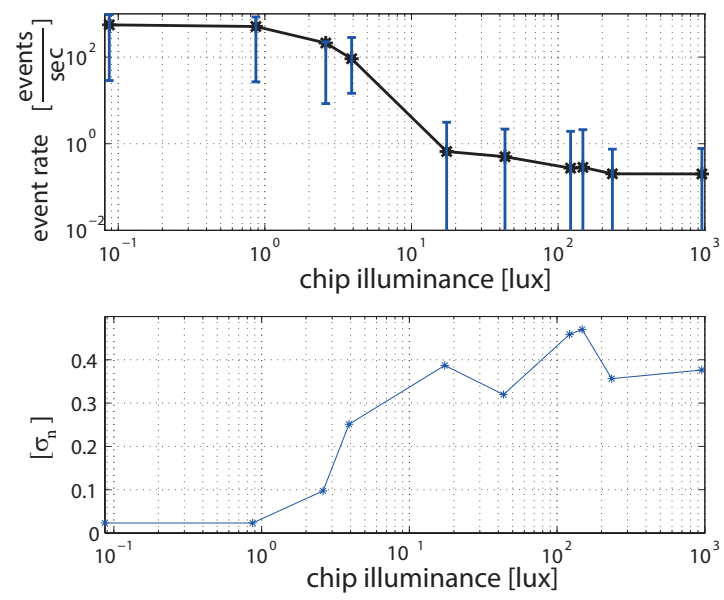

Fig. 19: Pixels temporal noise characterization. We measured the average output event rate for different uniform illumination levels (top). Error bars show the minimum and maximum values. On bottom, we display the normalized standard deviation $\sigma_{n}$ for each measurement. 
sults, pixel design was quite conservative because photodiodes spectral sensitivity was unknown beforehand. In future work, pixels size could be better optimized in terms of area. We believe that color change detectors could coexist with other vision sensors in the same chip to model part of the visual processing of the human retina.

\section{REFERENCES}

[1] T. Young, "Bakerian lecture: Experiments and calculations relative to physical optics," Philosophical Transactions of the Royal Society, vol. 94, pp. 1-16, 1804.

[2] J. E. Dowling, The Retina: An Approachable Part of the Brain, 2nd ed. The Belknap Press of Harvard University Press, 2012.

[3] K. Fukushima, Y. Yamaguchi, M. Yasuda, and S. Nagata, "An electronic model of the retina," Proceedings of the IEEE, vol. 58, no. 12, December 1970.

[4] M. Mahowald, An Analog VLSI System for Stereoscopic Vision. Kluwer, 1994.

[5] J. M. A. Olsson and P. Hafliger, "Two color asynchronous event photo pixel," in ISCAS, 2008, pp. 169-172.

[6] R. Berner and T. Delbruck, "Event-based pixel sensitive to changes of color and brightness," IEEE Transactions On Circuits and Systems I, vol. 58, no. 7, pp. 1581-1590, 2011.

[7] J. A. Leñero-Bardallo, D. Bryn, and P. Häfliger, "Bio-inspired asynchronous pixel event tricolor vision sensor," Biomedical Circuits and Systems, IEEE Transactions on, vol. 8, no. 3, pp. 345-357, June 2014.

[8] FOVEON, "http://www.foveon.com."

[9] G. de Graaf and R. Wolffenbuttel, "Smart optical sensor systems in cmos for measuring light intensity and colour," Sensors and Actuators, pp. 115-119, 1998.

[10] J. Tiffin and H. S. Kuhn, "Color discrimination in indurstry," Archives of Ophthalmology, vol. 28, no. 5, pp. 851-859, November 1942.

[11] Ł. Farian, J. A. Leñero-Bardallo, and P. Häfliger, "A bio-inspired aer temporal tri-color differentiator," IEEE Biomedical Circuits and Systems Conference (BioCAS), Lausanne, Switzerland, pp. 524-527, October 2014.

[12] — "Live demonstration: A bio-inspired aer temporal tri-color differentiator," IEEE Biomedical Circuits and Systems Conference (BioCAS), Lausanne, Switzerland, p. 171, October 2014.

[13] P. Häfliger, "A spike based learning rule and its implementation in analog hardware," Ph.D. dissertation, ETH Zürich, Switzerland, 2000, http:// www.ifi.uio.no/ hafliger.

[14] K. A. Boahen, "Point-to-point connectivity between neuromorphic chips using address events," IEEE Trans. Circuits Syst. II, vol. 47, no. 5, pp. 416-434, 2000.

[15] J. A. Leñero-Bardallo and P. Häfliger, "A dual-operation-mode bioinspired pixel," Circuits and Systems II: Express Briefs, IEEE Transactions on, vol. 61, no. 11, pp. 855-859, Nov 2014.

[16] P. Lichtsteiner, C. Posch, and T. Delbruck, "A $128 \times 128120 \mathrm{~dB} 15 \mu \mathrm{s}$ latency asynchronous temporal contrast vision sensor," IEEE Journal of Solid-State Circuits, vol. 43, no. 2, pp. 566-576, February 2008.

[17] R. Berner, T. Delbruck, A. Civit-Balcells, and A. Linares-Barranco, "A 5Meps \$100 USB 2.0 address-event monitor-sequencer interface," in ISCAS 2007, New Orleans, 2007, pp. 2451-2454.

[18] “jAER open source project," http://sourceforge.net/projects/jaer/.

[19] G. de Graaf, F. Riedijk, and R. Wolffenbuttel, "Color sensor system with a frequency output and an ISS or I2C bus interface," Sensors and Actuators, pp. 441-445, 1997.

[20] T. Delbruck and C. Mead, "Adaptive photoreceptor with wide dynamic range," in Circuits and Systems, 1994. ISCAS '94., 1994 IEEE International Symposium on, vol. 4, May 1994, pp. 339-342 vol.4. 